\title{
Methodology for determining critically important objects of energy systems from positions of ensuring energy security
}

\author{
Sergey Vorobev $^{1 *}$, Sergey Senderov ${ }^{1}$ \\ ${ }^{1}$ Melentiev Energy Systems Institute, 130 Lermontov str., Irkutsk, Russia
}

\begin{abstract}
An increase in the number of major accidents in energy systems in recent years has been due to the significant depreciation of fixed assets, and the lack of significant financial investments in their reconstruction. Large-scale accidents in energy systems resulting from the failure of the most important system objects entail significant, sometimes irreparable, damage to consumers in the form of large short deliveries of final types of energy. Thus, the identification of the most important facilities and their combinations in energy systems with the subsequent development of measures aimed at reducing the importance of such facilities is relevant today. The article reflects the main points of the comprehensive work on the search and identification of critical objects of the gas industry. The lists of these objects and their combinations, ranked by the degree of influence on consumers, are formed. Possible invariant measures aimed at reducing the importance of such objects are presented.
\end{abstract}

\section{Introduction}

The energy security of Russia and its regions concerns two main aspects:

- the need for long-term deficit-free provision of consumers with the required types of energy resources during the operation of the energy sector under normal conditions;

- creation of conditions for providing consumers with energy resources in emergency situations.

Consideration of the second aspect, first of all, requires the allocation of critical objects in the fuel and energy complex, i.e. those facilities, partial or complete failure of which can cause significant social and economic damage to the country.

The selection of the critical objects of fuel and energy complex is directly related to two major tasks:

- identification and neutralization of various kinds of threats to sustainable fuel and energy supply to consumers (including the threat of terrorist acts at the fuel and energy complex);

- early preparation of objects and systems of the fuel and energy complex for work during emergencies caused by the implementation of threats of various types.

Such work should obviously be carried out in terms of determining the critical objects for the main energy sectors separately, and then for the fuel and energy complex as a whole. To solve the set tasks at the level of sectoral energy systems, sufficiently detailed simulation mathematical models should be used. The use of such models should give results in terms of assessing the dependence of the performance of the relevant energy sectors on the operation of specific energy facilities.
Those objects on which such a dependence of the entire system is tangible and, moreover, critical, should be recognized as critical from the standpoint of ensuring the operability of the system as a whole. Precisely such objects should be primarily targeted by measures to ensure the survivability of the corresponding energy system. When analyzing the critical objects of the fuel and energy complex level, a specialized model apparatus can be used that adequately describes all aspects of the interrelated functioning of energy industries within a single fuel and energy complex from the standpoint of energy security. Such a model apparatus will make it possible to determine the total capabilities of the country's fuel and energy complex and the fuel and energy supply systems of specific regions to meet the needs of individual territories in various types of energy, which actually develop in various conditions (including emergency situations). At the same time, the own capabilities of the fuel and energy complex will be taken into account to compensate for the negative consequences of the loss of efficiency of the critical objects of various industries. First of all, these are the possibilities of interchangeability of various fuel and energy resources in the production of final types of energy and the possibility of diversifying energy sources. Only with the help of a model apparatus for relevant studies at the fuel and energy complex level can one get an idea of the potential list of the fuel and energy complex, i.e. those critical objects of the energy sectors, the negative consequences of the loss of working capacity of which cannot be compensated even with the indicated possibilities of the interconnected work of the

*Corresponding author: seregavorobev@isem.irk.ru 
energy systems within the framework of a single fuel and energy complex.

The solution to any complex research problem must start from the bottom of the components of a large system. As for the Russian fuel and energy complex as a whole, for the European part of Russia the main type of fuel is natural gas. In the country as a whole, the share of gas in the balance of boiler and furnace fuel (mainly fuel for thermal power plants) is about $77 \%$. In a significant part of the regions, its share in the fuel balance exceeds $90-95 \%$, and sometimes it reaches $99 \%$.

Thus, an urgent task today is to develop a methodology for determining critical objects of energy systems from the standpoint of ensuring energy security and the corresponding definition of such objects and their combinations in energy systems with the subsequent development of measures aimed at reducing the importance of such objects.

\section{Analysis of published works in the field of search and identification of critical objects of energy systems}

The analysis of the published works has shown that today research is being actively conducted concerning the identification of critical objects of energy systems.

In works [1, 2], the authors analyzed the gas transmission network in order to determine its most important elements. The methodological approaches applied in this case are based on topological network analysis with an emphasis on the study of issues of reliability and controllability. This analysis makes it possible to quantitatively assess the reliability of the gas transmission network and determine the role of each component of the network in different time slices. A real gas transmission network in several EU countries is considered as an example. The article presents the results of the analysis of such a critical infrastructure, shows the need to take into account physical characteristics, such as restrictions on the throughput of gas pipelines. To assess the consequences of the implementation of negative external influences on the possibilities of gas supply to consumers, a special flow model has been developed. Vulnerability analysis is performed from three perspectives: global vulnerability analysis, demand reliability, and critical analysis of gas pipelines. The global vulnerability analysis is carried out taking into account possible disturbances in the operation of gas sources and transport. Demand reliability analysis assesses the ability of consumers to withstand external influences on them. The critical analysis of gas pipelines considers the impacts on specific gas pipelines.

Work [3] presents a method for defining and ranking critical components and component sets in technical infrastructures. The criticality of a component or set of components is defined as the vulnerability of a system to failure when a particular component or set of components fails. The question is also devoted to the problem of multiple simultaneous failures, and even with synergistic consequences. The proposed method solves this problem. An analysis of the power distribution system in a Swedish municipality is presented as an example of this method.

In [4], a comprehensive model is proposed for assessing the impact of the interdependence of electrical and gas systems on the reliability of energy supply to consumers. The operating mode of the gas network is modeled using restrictions on the operation of the main elements. Gas supply restrictions can affect the change in the operating modes of the electric power industry. This is shown by illustrative examples given by the authors.

In [5], through the analysis of the possible impacts of the integrated gas and electric network, it is shown that failures of the gas supply system can be considered more decisive for the integrated power supply system than failures in the power supply subsystem itself. Accordingly, the authors paid attention to possible control actions aimed at minimizing the negative impact of failures in the gas supply system. At the same time, such an approach is possible provided that the basis of electricity generation is made up of power plants using natural gas.

In works [6-8], the authors come closest to the definition of critical objects of the energy system, in this case, the gas transmission network. At the same time, they assign different indices to different objects of the system in a complex that determine the vulnerability of the system in case of disruption of the operation of this object.

Taking into account the previously gained experience and based on the analysis of research carried out in the world at the present time in $[9,10]$ formulated a methodology for the formation of lists of critical objects from the standpoint of ensuring the operability of these systems on the example of the gas industry in Russia.

\section{The main provisions of the methodology for determining the critical objects of gas industry}

Significant gas reserves are concentrated in Russia (the Yamal and Gydan peninsulas, the shelf of the Barents and Kara Seas). The country has an extensive system of main gas and oil pipelines and a complex geographically distributed system of fuel and energy supply, covering the entire territory of Russia. The existing territorial structure of the Russian gas supply system determines its significant shortcomings. For example, the European part of the country is not provided with its own reserves of fuel and energy resources. It mainly uses natural gas, more than $90 \%$ of which is produced in one gasproducing region (Nadym-Pur-Tazovsky district of the Tyumen region). This area is located 2-2.5 thousand $\mathrm{km}$ from the places of the main gas consumption. Thus, practically all Russian gas is transported over long distances through the systems of main gas pipelines, which have a large number of mutual intersections and bridges; moreover, the lines of powerful main gas pipelines are often laid at a short distance from each other. Currently, in the gas transmission system of Russia, more than 20 potentially dangerous for the 
functioning of the system of intersections of main gas pipelines can be noted. The most significant of them is located almost at the very outlet of gas from its main fields: Urengoyskoye and Yamburgskoye. Disruption of the operation of such an intersection of gas flows can lead to an almost complete (90\%) limitation of gas needs at the national level as a whole.

The consequences of the implementation of various emergency situations in power systems with large-scale negative manifestations of natural and climatic processes, for example, abnormally cold periods in winter with a peak increase in the need for additional volumes of fuel, can be much more severe. In this case, the extremely increased demand for fuel may manifest itself not only in one single region. Most likely, this situation will be typical for a single climatic zone or several neighboring regions. In particular, this issue is relevant for the territories of the European part of the country characterized by a high share of natural gas consumption, because in such regions, the share of their own fuel and energy resources in their fuel balance is usually low.

With this in mind, as a first step, research was carried out using the example of the gas industry:

- developed an algorithm for identifying the critical objects of a specific system;

- an assessment of the role of specific critical objects in ensuring the operability of a specific energy system in the context of the implementation of various kinds of emergency situations;

- a list of measures was formed to minimize the negative consequences of a decrease in the level of performance of each selected critical objects of the energy system under consideration;

- a substantiation of the list of invariant measures to minimize the negative consequences from the action of various kinds of emergency situations on the selected critical objects of the considered power system was carried out, taking into account possible simultaneous combinations of emergency situations at different objects.

\section{Mathematical formulation of the problem of identifying critical objects}

When developing this methodology, to determine the critical objects themselves, and to search for critically important combinations of objects, were used the flow model, which is the core of the "Russian Oil and Gas" software, to determine the critical objects itself and to search for critical combinations of objects [11-15]. The use of this "Russian Oil and Gas" software allows user to determine the degree of satisfaction of gas needs within the country and ensure export supplies. In addition, the "Russian Oil and Gas" software allows user to identify bottlenecks - sections of network that in some cases limit the production capabilities of the system.

The flow distribution model in the Unified Gas Supply System of Russia in the "Russian Oil and Gas" software is designed to assess the production capabilities of the Unified Gas Supply System of Russia in conditions of various kinds of disturbances. The purpose of such studies is to minimize gas deficits at the consumption sites. The Unified Gas Supply System of Russia in the model is represented as a set of three subsystems: gas sources, main gas transport network and consumers.

When solving the problem of estimating the state of a system after a perturbation, the criterion of the optimality of the distribution of flows is the minimum gas deficit in the consumer with minimum costs for delivering gas to consumers. This problem can be solved by finding the maximum flow through the network, followed by minimizing the cost of gas delivery to consumers [16]. The mathematical formulation of this problem is described in [17].

In the flow distribution model in the Unified Gas Supply System of Russia, as already mentioned, the Basaker-Gowen algorithm is used to calculate the maximum flow of minimum cost, which as a result allows you to determine the possible level of gas consumer satisfaction. As a result of the implementation of various emergency situations, a gas shortage among consumers may occur due to a lack of flow capacity in certain sections of gas pipelines. Bypassing such narrow or limiting production possibilities of the system's places, in acceptable volumes, will allow reducing the gas shortage arising in the situation under consideration by consumers.

An integrated approach to solving the assigned tasks along the entire Unified Gas Supply System technological chain allows obtaining an overall assessment of the production capabilities of the entire system under extreme conditions. The result of solving the problem is to determine the possibilities of satisfying consumers with network gas with the identification of volumes of possible undersupply of gas to consumption nodes in a particular emergency situation. Based on these results, it is possible to obtain a list of facilities, as well as a list of combinations of facilities in the gas industry, the termination of which will lead to a potential shortage of gas in the network. We rank this list by the relative magnitude of the gas deficit in the network. By cutting off objects, the withdrawal of which will lead to a potential shortage of gas in the network less than the assigned value, for example, $5 \%$, it is possible to obtain a list of the critical objects of the gas industry. Such a list should also be ranked according to the degree of impact on network performance. The same mechanism applies to the procedure for determining critical combinations of gas facilities.

\section{Identification of critical objects In gas industry}

The design scheme of the Unified Gas Supply System used in this work takes into account all the main features of the functioning of the Unified Gas Supply System of Russia and contains:

- 378 nodes, including: 28 gas sources; 64 gas consumers (constituent entities of the Russian Federation); 24 
underground gas storage facilities; 266 nodal compressor stations;

- 486 arcs representing the main gas pipelines and branches to the gas distribution networks.

Relevant studies were carried out on the model of the Russian gas industry presented above. The initial conditions for the calculations are as follows: the average day of maximum gas consumption in the network, based on statistics on gas consumption by region in January [18-20]. On such days, the operation of the network can be considered extremely intense relative to the average annual load. The total gas flow through the network on such a day, taking into account export supplies, amounted to approximately 2,250 million $\mathrm{m} 3$. The results of these studies have shown that potential gas shortages among consumers will be observed when 441 facilities of the Russian gas industry are shut down (242 nodes and 199 arcs of the network computational graph). The threshold of being included in the list of critical objects with a potential gas shortage (total in 5\% of the total gas demand) in conditions of shutdown of one of these facilities was crossed by 61 facilities. These objects were included in the list of the critical objects of the federal level for the gas industry. Among these objects there are 25 arcs between nodal compressor stations and 36 nodes, including 30 nodal compressor stations, 5 head compressor stations at the outlets from large gas fields and one underground storage facility. Information on the calculated values of the relative gas shortages in the network when specific nodes and arcs are turned off in a form ranked by the degree of gas deficit reduction is presented in Table. 1 (the real names of the Unified Gas Supply System of Russia facilities in this article are replaced with conventional numbers).

Table 1. Estimated relative gas shortages in the network on the most intense day in January 2017

\begin{tabular}{|c|c|c|}
\hline$\#$ of object & Object type & Gas shortages, $\%$ \\
\hline $1,2,3,4$ & Node & 21 \\
\hline $5,6,7$ & Arc & 21 \\
\hline 8 & Node & 19 \\
\hline $9,13,14$ & Arc & 16 \\
\hline $10^{*}, 11,12,15$ & Node & 16 \\
\hline 16 & Arc & 12 \\
\hline $17,18,19,22,23$ & Node & 10 \\
\hline 20,21 & Arc & 10 \\
\hline 24 & Node & 9 \\
\hline $25,26,28^{*}$ & Node & 8 \\
\hline 27,29 & Arc & 8 \\
\hline $31,33,35,37,39,41$ & Arc & 7 \\
\hline $30^{*}, 32,34,36,38$, & Node & 7 \\
\hline 40 & Arc & 6 \\
\hline $42,48,50$ & Node & 6 \\
\hline $43^{*}, 44^{*}, 45,46^{* *}, 49,51$ & Arc & 5 \\
\hline $52,55,56,59,60$ & Node & 5 \\
\hline $53,54,57,58,61$ & . & 7 \\
\hline
\end{tabular}

* - the node refers to production targets, i.e. to the gas compressor station at the exits from the fields.

** - the node refers to underground gas storage facilities (UGS).
From the data table. 1 that when each of the first eight objects of the ranked list of the critical objects of the gas industry at the federal level is disconnected, the relative gas deficit in the system can be about $20 \%$ of the required total supply. Disabling each of the following 15 objects can result in a $10-16 \%$ system flow restriction. Disconnection of all other objects from the list of critical objects can provoke a relative shortage of gas in the system within 5-9\% [10].

\section{Identification of critical combinations of gas facilities}

After the above list of the gas industry critical objects was formed from the standpoint of ensuring its operability, calculations were carried out on this calculation scheme to determine the critical combinations of UGSS facilities with each individual critical object. In addition, the next step was to simulate the process of "breaking" bottlenecks aimed at minimizing gas shortages among consumers by increasing its flow through individual sections of the network.

The criterion for the inclusion of each combination of gas industry facilities with a specific critical object in the list of critical combinations will be the difference in the relative total gas deficit among consumers when the i-th critical object stops working and the combination of the $i$-th critical object with the j-th object of the settlement network stops working:

$$
\begin{gathered}
\Delta Q_{i j}=Q_{i j}-Q_{i,} \quad i=1, \ldots, K ; j=1, \ldots, N ; i \neq j \\
\Delta Q_{i j} \geq \delta
\end{gathered}
$$

$\mathrm{Q}_{\mathrm{ij}}$ - is the total relative gas deficit among consumers caused by the termination of the operation of the combination of the $\mathrm{i}$-th critical object and the $\mathrm{j}$-th facility of the settlement network. $\mathrm{Q}_{\mathrm{i}}$ - is the total relative gas deficit among consumers caused by the termination of the $\mathrm{i}$-th critical object, $\delta$ is the limitation of the relative increment of the total gas deficit at consumers to be included in the list of critical combinations of gas facilities.

Calculations show that more than 15 thousand combinations of other facilities with dedicated air cooling systems lead to an increase in gas deficit among consumers. Based on considerations of the acceptability of the expert analysis, we will limit the value of $\delta$ within $5 \%$. Because of calculating in pairs all 61 critical objects of UGSS with the rest of the system objects (in total, more than 61 thousand pairs were analysed for their simultaneous shutdown). 630 pairs were obtained, consisting of one critical object and another object of the gas industry, the failure of which can lead to the emergence more than $5 \%$.

It can be noted that the termination of the operation of critical combinations of UGSS facilities can lead to an increase in gas shortages to consumers by an average of $8-10 \%$ of the required gas volumes compared to the shortage caused by the termination of the operation of the corresponding UGSS facilities. In some cases, this increase can reach $20 \%$. 
As for measures to bypass bottlenecks, in situations with the termination of operation of the considered critical combinations of facilities, such measures lead to a relatively insignificant decrease in the total gas deficit among consumers (on average by 2-3\%). This fact additionally confirms the high importance of identifying these combinations. [10].

\section{Application of the method for determining critical elements in the networks of technical infrastructures in the search for critical objects of the gas industry}

Determining critical elements is usually a straightforward task when considering only single failures. When considering multiple concurrent failures, this task can become much more complex.

It is especially difficult to identify critical groups of elements with a so-called synergistic effect. In this context, the synergistic effect means that the negative consequences of the failure of the group as a whole are higher than the total impact of individual failures of the elements included in the group. In other words, the failure of a group of two elements with serious negative consequences can have a synergistic effect if the failure of each of the elements does not in itself cause any significant consequences.

The method for determining critical elements in networks of technical infrastructures [21] facilitates the identification and ranking of such groups of elements (as well as groups of elements, the failure of which does not give a synergistic effect). Found critical elements or sets of elements can then be studied in more detail using probabilistic methods of risk analysis [22].

The essence of the above method is to study the sets of failures, each of which represents a set of faulty elements, has only one negative effect on the system, and is characterized by a size that specifies the number of elements whose failure occurs simultaneously.

The number of elements whose failure occurs simultaneously is a set of failures $n$, its size is chosen by the researcher depending on the total number of system elements t. However, for practical reasons, $\mathrm{n}$ should not exceed 3 or 4 , since the number of possible sets of failures equal to $t ! /((t-n) ! * n !)$. Grows rapidly as $n$ increases, which inevitably leads to an increase in the computation time. In addition, if we talk about a real power system, such as the UGSS, the probability of a simultaneous failure of a large number of independent elements of this system is very small.

The ranking of the sets of failures is carried out in accordance with the magnitude of their synergistic effects.

Using the design scheme presented above, the search for the critical objects in the UGSS was carried out using the method for determining the critical elements in the networks of technical infrastructures. As a result of calculations, 5 sections of main gas pipelines were obtained, which are critical both from the point of view of the maximum gas shortage among consumers and in terms of their contribution to the synergistic effect. Disruption of the functioning of these sections will lead to a significant gas shortage among consumers, from 15 to $21 \%$ in total throughout the entire system. All these factors make it possible to classify these sections of main gas pipelines as the critical objects of the gas industry.

The application of the method for determining critical elements in the networks of technical infrastructures in the search for critical objects clearly shows that the disruption of the functioning of several unconnected sections of the main gas pipelines, as well as the disruption of the functioning of the intersection of the main gas pipelines, will most likely cause more harm to the system than the disruption of the functioning of one section of the main gas pipelines. Taking this fact into account, we can talk about the greater importance of the sections of main gas pipelines as critical objects with a high value of the criticality index [23].

\section{Determination of the most important combinations of gas facilities}

All possible major combinations of gas facilities were identified and analysed. The most important combination of objects within the framework of this study means a pair of unconnected, independent objects, the failure of which can lead to a significant gas shortage among consumers. At the same time, the objects under consideration should not be included in the list of critical objects, or in the list of critical combinations of objects.

Taking into account the previously obtained 61 critical objects and 630 pairs of critical combinations, calculations were carried out for the pairwise disconnection of all other objects of the design scheme, followed by "uncovering" bottlenecks - by taking measures aimed at minimizing gas shortages among consumers. These calculations were carried out using a software package [24] that reflects in detail the functioning of the Russian gas transmission network and allows simulating various conditions for the functioning of its facilities, including a complete shutdown. The calculations were carried out using the parallel computation methodology in [25].

As a result, out of the 207690 pair combinations obtained, 2865 object pairs were selected, the failure of which leads to a total gas deficit in the system of $5 \%$ or more. After solving the problem of bypassing the bottlenecks, 2555 pairs of objects remained.

Table 2 shows 20 combinations of facilities, the failure of which can lead to a gas shortage in the system as a whole $10 \%$ or more. 
Table 2. Combinations of Unified Gas Supply System of Russia objects, failure of which will lead to a maximum gas shortage in the system as part of the study

\begin{tabular}{|c|c|c|c|}
\hline $\begin{array}{c}\text { № of } \\
\text { pair }\end{array}$ & $\begin{array}{c}\text { Object type } \\
\text { №1 }\end{array}$ & $\begin{array}{c}\text { Object type } \\
\text { №2 }\end{array}$ & $\begin{array}{c}\text { Gas shortage, } \\
\%\end{array}$ \\
\hline 1 & CS & CS* & 11 \\
\hline 2 & CS* & CS & 11 \\
\hline 3 & Arc & CS $^{*}$ & 11 \\
\hline 4 & Arc & CS $^{*}$ & 11 \\
\hline 5 & CS & CS & 11 \\
\hline 6 & CS & CS* & 11 \\
\hline 7 & CS & CS* & 10 \\
\hline 8 & Arc & CS & 10 \\
\hline 9 & Arc & Arc & 10 \\
\hline 10 & Arc & CS & 10 \\
\hline 11 & CS & CS & 10 \\
\hline 12 & CS & CS & 10 \\
\hline 13 & Arc & CS & 10 \\
\hline 14 & Arc & CS & 10 \\
\hline 15 & CS & CS* & 10 \\
\hline 16 & CS & CS* & 10 \\
\hline 17 & Arc & CS* & 10 \\
\hline 18 & Arc & CS* & 10 \\
\hline 19 & Arc & Arc & 10 \\
\hline 20 & Arc & CS & 10 \\
\hline
\end{tabular}

When analyzing the table 2 , it is necessary to highlight an object - one nodal compressor station (CS*), which is not included in the list of critical objects. CS* is present in 10 combinations from the table 2 . In addition, this CS* is present in $25 \%$ of all combinations leading to a total gas deficit of the system as a whole of $5 \%$ or more.

In general, the following should be noted from the results of the study. Violation of the functioning of the most important combination of objects can lead to a significant gas shortage among consumers (5-15\%).

In this situation, measures to bypass bottlenecks lead to a slight decrease in the gas deficit in the system as a whole (by an average of 2-3\%). This fact confirms the high importance of the identified combinations. It is worth noting that in the framework of this study, as a result of bypassing bottlenecks, the number of possible most important combinations of objects was reduced by $10 \%$.

The results of this study showed that in the modern configuration of the UGSS, situations are possible when, in the event of a failure of a pair of network objects that are not critical object, the total gas deficit among consumers can reach $15 \%$ of the total gas demand [26].

\section{Identification of especially significant objects of the gas industry}

In addition to the critical objects, there is a significant number of facilities in the rather complex and ramified gas transportation system of Russia, the termination of which can lead to significant restrictions on gas supplies to a particular region. It was proposed to name these objects as especially significant objects of the UGSS.
They were identified in the course of a special study [26], and a ranked list of them was formed. The list of especially significant objects UGSS by the number of objects exceeds the list of critical objects UGSS and fully includes all of them.

As a result of model studies that simulate the operation of the Russian gas industry in the conditions of alternate shutdowns of each of the facilities of the Russian gas industry, 193 UGSS especially significant objects were identified, the failure of which would lead to a gas deficit in any region in the amount of $10 \%$ or more. Among these objects there are 94 nodes of the gas transmission network and 99 arcs. At the same time, the total number of UGSS facilities participating in the calculation is 1004 . Thus, $19 \%$ of them are included in the list of UGSS facilities.

The results of the study showed that for some regions, the termination of each facility from the list of especially significant objects affecting gas supplies to a given region leads to a $100 \%$ gas deficit. That is, for example, the termination of the operation of any of the 33 especially significant objects affecting the process of gas supply to the Kirov region will inevitably lead to a complete cessation of gas supply to this region.

Further, scenarios were calculated for the simultaneous shutdown of combinations of such objects by 2 . Research showed that when searching for the most significant, from the point of view of consumer satisfaction, combinations of UGSS objects, 1,789 thousand combinations were analyzed, respectively, the same number of calculations were carried out. As a result, 18,528 combinations of UGSS facilities were found, the failure of which could cause a $10 \%$ or more relative gas shortage in at least one of the regions under consideration.

The identification of especially significant UGSS facilities and especially significant combinations of UGSS facilities and the formation of their lists is the next step after identifying the critical objects of the gas industry on the way to form a list of especially vulnerable regions from the point of view of fuel supply in the context of various emergencies in the gas industry. Taking into account in these studies the concept of vulnerability of the fuel supply system of a particular region makes it possible to draw conclusions about the need to plan measures to reduce this indicator in a number of regions.

\section{Conclusion}

The article reflects the main points of comprehensive work on the search and identification of critical objects of the gas industry, which form the basis of the methodology for determining critical objects of energy systems from the standpoint of ensuring energy security. The results of research are presented to determine:

- critical objects of the gas industry, 61 facilities, the failure of which can lead to a gas deficit of $5 \%$ or more throughout the system; 
- critical combinations of gas industry facilities, 630 pairs of facilities, total gas deficit in case of a pair failure - by $5 \%$ or more from more than one critical object;

- the most important combinations of gas objects, 2555 pairs of facilities, failure of which leads to a total gas deficit in the system of $5 \%$ or more;

- especially significant objects of the gas industry, 193 facilities, the failure of which will lead to a gas deficit in any region in the amount of $10 \%$ or more;

- especially significant combinations of gas industry objects, 18,528 combinations of facilities, the failure of which can cause a $10 \%$ or more relative gas shortage in at least one of the regions under consideration.

Conclusions are drawn about the necessity and feasibility of searching for and determining these objects, with the subsequent development of invariant measures aimed at reducing their significance.

One of the possible directions for the development of this study is shown, associated with deepening into the problems of vulnerability of fuel supply systems in regions and their dependence on natural gas supplies.

The study was carried out in the framework of the draft state assignment III.17.5.1 (reg. No. AAAA-A17117030310451-0) of basic research of the SB RAS and RFBR grant No. 20-08-00367.

\section{References}

1. Han F. \& Zio E. \& Kopustinskas V. \& Praks P. Quantifying the importance of elements of a gas transmission network from topological, reliability and controllability perspectives, considering capacity constraints. In book: Risk, Reliability and Safety: Innovating Theory and Practice, 2016, pp. 2565-2571. DOI 10.1201/9781315374987-389,

2. Su H., Zio E., Zhang J. Li X. A systematic framework of vulnerability analysis of a natural gas pipeline network. Reliability Engineering \& System Safe-ty, Volume 175, July 2018, Pages 79-91 https://doi.org/10.1016/j.ress.2018.03.006

3. Jonsson H., Johansson J., Johansson H. Identifying critical components in technical infrastructure networks. Proceedings of the Institution of Mechanical Engineers, Part O: Journal of Risk and Reliability. 2008. Vol. 222. No. 2. P. 235-243. https://doi.org/10.1243\% 2F1748006XJRR138

4. Li T., Eremia M., Shahidehpour M., Interdependency of natural gas network and power system security, IEEE Transactions on Power Systems, 2008, vol. 23, 1817-1824. https://doi.org/10.1109/ TPWRS.2008.2004739

5. Dokic S.B., Rajakovic N.Lj. Security Modelling of Integrated Gas and Electrical Power Systems by Analyzing Critical Situations and Potentials for Performance Optimization. Energy (2018), doi: 10.1016/j.energy. 2018.04.165.

6. V. Kopustinskas, P. Praks. Identification of the main contributors to the security of supply in a gas transmission network. In: Probabilistic Safety Assessment and Management PSAM 14, September 2018, Los Angeles, USA.
7. V. Kopustinskas, P. Praks. Bottleneck analysis of the gas transmission network using ProGasNet simulator. In Luca Podofillini et al. (ed.), Safety and Reliability of Complex Engineered systems; Proc. of ESREL'2015, Zurich 7-10 September 2015. Leiden, CRC Press/Balkema.

8. P. Praks, V. Kopustinskas, M. Masera. Probabilistic modelling of security of supply in gas networks and evaluation of new infrastructure. Reliability Engineering and System Safety 144:254-264, (2015).P. Praks, V. Kopustinskas, M. Masera. "MonteCarlo based reliability and vulnerability assessment of a natural gas transmission system due to random network component failures", Sustainable and Resilient Infrastructure, Vol. 2(3), pp.97-107, (2017).

9. Senderov S., Edelev A. Formation of a list of critical facilities in the gas transportation system of Russia in terms of energy security. Energy, 2019, doi:10.1016/j.energy.2017.11.063.

10. S.M. Senderov, S.V. Vorobev, Approaches to the identification of critical facilities and critical combinations of facilities in the gas industry in terms of its operability. Reliability Engineering \& System Safety, Volume 203, 107046, 2020, doi: 10.1016/j.ress.2020.107046.

11. Senderov S.M., Rabchuk V.I., Edelev A.V. Features of the formation of a list of critical objects of the gas transmission network of Russia, taking into account the requirements of energy security and possible measures to minimize the negative consequences of emergencies at such facilities / Izvestiya RAN. Energetika, 2016, No. 1, p. 70-78.

12. Voropai N.I., Senderov S.M., Edelev A.V. Detection of "bottlenecks" and ways to overcome emergency situations in gas transportation networks on the example of the European gas pipeline network. Energy, 2012, doi:10.1016/j.energy.2011.07.038.

13. Edelev A.V., Enikeeva S.M., Senderov S.M. Information support in the study of the functioning of large pipeline systems / Computational technologies. 1999. - Volume 4, No. 5. - P. 30 - 35.

14. Vorobev S.V., Edelev A.V. Methodology for determining bottlenecks in the operation of large pipeline systems / Software products and systems. 2014. - No. 3. - P. 174 - 177.

15. Khramov A.V., Enikeeva S.M., Khrustaleva N.M. et al. Software and information support for solving the problems of survivability of the Unified Gas Supply System of the USSR // in Methods and models for studying the survivability of energy systems, Novosibirsk: Nauka, Sib. dep, 1990, p. 86-91.

16. Ford L.R., Fulkerson D.R. Flows in Networks / Princeton University Press, Princeton, New Jersey, 1962, $276 \mathrm{p}$.

17. S. Vorobev, A. Edelev, E. Smirnova, RSES 2017. E3S Web Conf. Vol. 25, DOI 10.1051 / e3sconf / 20172501004. (2017).

18. Export of the Russian Federation of the most important goods in 2012 - 2019 (according to the Federal Customs Service of Russia) http://customs.ru/ index.php?option=com _ newsfts\&view =category\&id= 52\&Itemid $=1978 \&$ limitstart $=60$. 
19. InfoTEK Monthly oil and gas magazine. No. 1, 2019, p. 154.

20. Ministry of Energy of the Russian Federation. Statistics. http://minenergo.gov.ru/activity/statistic.

21. Jonsson H., Johansson J., Johansson H. Identifying critical components in technical infrastructure networks. Proceedings of the Institution of Mechanical Engineers, Part O: Journal of Risk and Reliability. 2008. Vol. 222. No. 2. P. 235-243.

22. Johansson J., Hassel H., Zio E. Reliability and vulnerability analyses of critical infrastructures: comparing two approaches in the context of power systems. Reliability Engineering \& System Safety. 2013. No. 120. P.27-38.

23. Vorobev S., Edelev A., Smirnova E. Search of critically important objects of the gas industry with the method of determining critical elements in networks of technical infrastructures, Methodological Problems in Re-liability Study of Large Energy Systems (RSES 2017). E3S Web Conf. Volume 25, 2017. doi: 10.1051/e3sconf/20172501004.

24. Feoktistov A., Gorsky S., Sidorov I., Kostromin R., Edelev A., Massel L. Orlando Tools: Energy Research
Application Development through Convergence of Grid and Cloud Computing / Communications in Computer and Information Science. 2019. Vol. 965. P. 289-300.

25. Irkutsk Supercomputer Center SB RAS. URL: http://hpc.icc.ru

26. Vorobev S., Smirnova E. Search of the most important combinations of gas industry objects from the positions of system operability, Rudenko International Conference "Methodological problems in reliability study of large energy systems" (RSES 2019), E3S Web Conf. Vol. 139, 2019. doi: 10.1051/e3sconf/201913901016.

27. Senderov S.M., Smirnova E.M., Vorobev S.V. Approaches to assessing the vulnerability of fuel supply systems to gas-consuming regions of Russia in the context of the shutdown of especially significant facilities in the gas industry / Izvestia RAS. Energetika, No. 1, 2020, p. 82-91. 